\title{
High-dose-rate intraluminal brachytherapy prior to external radiochemotherapy in locally advanced esophageal cancer: preliminary results
}

\author{
Afsaneh Maddah Safaei, MD!, Reza Ghalehtaki, MD!, Nezhat Khanjani, MD², Borna Farazmand, MD!, \\ Mohammad Babaei, MD!, Ebrahim Esmati, MDl \\ IRadiation Oncology Research Center (RORC), Cancer Institute, Tehran University of Medical Sciences, Tehran, Iran, ${ }^{2}$ Department of Radiation \\ Oncology, Namazi Hospital, Shiraz University of Medical Sciences, Shiraz, Iran
}

\begin{abstract}
Purpose: Dysphagia is a common initial presentation in locally advanced esophageal cancer and negatively impacts patient quality of life and treatment compliance. To induce fast relief of dysphagia in patients with potentially operable esophageal cancer high-dose-rate (HDR) brachytherapy was applied prior to definitive radiochemotherapy.

Material and methods: In this single arm phase II clinical trial between 2013 to 2014 twenty patients with locally advanced esophageal cancer (17 squamous cell and 3 adenocarcinoma) were treated with upfront 10 Gy HDR brachytherapy, followed by 50.4 Gy external beam radiotherapy (EBRT) and concurrent chemotherapy with cisplatin/5-fluorouracil.

Results: Tumor response, as measured by endoscopy and/or computed tomography scan, revealed complete remission in 16 and partial response in 4 patients (overall response rate 100\%). Improvement of dysphagia was induced by brachytherapy within a few days and maintained up to the end of treatment in $80 \%$ of patients. No differences in either response rate or dysphagia resolution were found between squamous cell and adenocarcinoma histology. The grade 2 and 3 acute pancytopenia or bicytopenia reported in 4 patients, while sub-acute adverse effects with painful ulceration was seen in five patients, occurring after a median of 2 months. A perforation developed in one patient during the procedure of brachytherapy that resolved successfully with immediate surgery.

Conclusions: Brachytherapy before EBRT was a safe and effective procedure to induce rapid and durable relief from dysphagia, especially when combined with EBRT.

J Contemp Brachytherapy 2017; 9, 1: 30-35 DOI: https://doi.org/10.5114/jcb.2017.65147
\end{abstract}

Key words: brachytherapy, chemoradiotherapy, esophageal cancer, intraluminal, quality of life.

\section{Purpose}

Esophageal cancer is known to have a high prevalence in Asian continent, including Iran. In other words, the world highest-risk area stretches from northern Iran through the central Asian republics to north-central China (often referred to as the "esophageal cancer belt") [1]. According to Iranian national cancer registry, esophageal cancer is reported to be the second and fourth most common cancer among males and the females, respectively [2]. Unfortunately, the disease is asymptomatic at early stages, and locally advanced disease is a usual presentation. Thus, despite developments in the treatment of esophageal carcinoma, the outcomes remain poor. Surgery following neoadjuvant radiochemotherapy is the preferred curative treatment for operable patients. However, a substantial number of patients are unfit for surgery. Concurrent chemoradiotherapy is the present accepted standard of care for patients who are not amenable to surgery [3]. In order to improve outcomes, radiotherapy dose escalation was being increasingly reported to improve the outcomes in esophageal cancer [4]. However, dose escalation with external radiotherapy accompanies sever acute and late morbidities. Intraluminal brachytherapy permits delivering escalated doses to a localized area of the esophagus with relative sparing of surrounding normal structures [5]. This technique may be used alone or as a boost in combination with external beam radiotherapy (EBRT). Brachytherapy has even be considered as an alternative to stent placement for palliation of dysphagia, particularly when the extent of extraluminal disease is limited and long-term palliation is likely to occur. Although stenting relieves the dysphagia immediately, the effect of brachytherapy lasts longer [6], and patients experience a reduced amount of further dilatation attempts and better quality of life [7].

\footnotetext{
Address for correspondence: Ebrahim Esmati, MD, Department of Radiation Oncology, Cancer Institute, Tehran University of Medical Sciences, Keshavarz Blvd., Tehran, Iran, phone/fax: +98 21 61192518, 
Esophageal brachytherapy involves intraluminal placement of a radioactive source into with an orogastric applicator. As a rule, the diameter of the applicator should be 0.6 to $1.0 \mathrm{~cm}$. Narrower applicators may deliver significantly higher doses to the mucosa resulting in important acute and late treatment-related toxicities; larger catheters pose the risk of abrasion, perforation, or combination thereof. The dose of radiation is generally prescribed to a site $1 \mathrm{~cm}$ from the midsource or midwell position. An area covering 1 to $2 \mathrm{~cm}$ proximal and distal to the lesion is treated [8]. According to high incidence of locally advanced esophageal cancer in Iran with dysphagia as the main symptom leading to weight loss and treatment noncompliance, we applied brachytherapy before EBRT to induce rapid tumor volume reduction and subsequent relief of dysphagia in a prospective study.

\section{Material and methods}

\section{Study design}

This single arm phase II non-randomized clinical trial study prospectively evaluated the role of high-dose-rate (HDR) intraluminal brachytherapy (ILBT) followed by chemoradiation in patients with potentially operable esophageal cancer between 2013 and 2014. They were planned to receive concurrent chemoradiotherapy two weeks after ILBT with a curative intent. The study was confirmed by institutional review board, received approval of university ethical committee of researches, designed and conducted in agreement with declaration of Helsinki, and all subjects were provided informed consent prior to enrollment.

\section{Inclusion and exclusion criteria}

We enrolled patients with pathologically confirmed locally advanced (T3/T4 or node-positive) esophageal carcinoma with compliant of moderate to severe dysphagia with no age limitation. Patients with one of following situations were excluded from the study; severe esophageal obstruction, esophageal fistula, Karnofsky performance status $<70$, cervical or gastroesophageal junction location, multiple/skip lesions, tumor length $>10 \mathrm{~cm}$, metastatic disease at presentation or recurrent disease.

\section{Pretreatment evaluation}

Primary work up included history and physical exam, blood lab tests, esophagogastroduodenoscopy without dilatation, chest and abdominopelvic computed tomography scan, upper endoscopic ultrasonography (EUS). In addition, barium swallow was done 2 days prior to initiation of ILBT for treatment planning. In patients, whose tumors was not recognized with barium, the location of the tumor was identified using the initial endoscopic and computed tomography (CT) findings.

\section{Intraluminal brachytherapy}

The planning for brachytherapy was performed using barium study preferably or simulation CT scan and esophagogastroduodenoscopy when barium study was normal. Superior and inferior borders of the length to be treated by ILRT were marked on the patient's chest to facilitate the ILRT planning. The total prescribed dose was 10 Gy in 2 fractions one week apart (BED = 15 Gy). The reference point for dose prescription was $1 \mathrm{~cm}$ away from the central axis. A $2 \mathrm{~cm}$ cranial and caudal margin was added to the superior and inferior borders of the gross tumor volume (GTV), providing that total treated length not to exceed $10 \mathrm{~cm}$. The treatment was delivered on a MicroSelectronHDR remote after-loading device (Nucletron, an Elekta company, Elekta AB, Stockholm, Sweden) with a $6 \mathrm{~mm}$ external diameter applicator.

\section{Concurrent radiochemotherapy}

Two weeks after completion of ILBT, the patients underwent EBRT that was delivered using a three-dimensional conformal radiotherapy (3DCRT) technique with 18 megavolt $X$-ray photons via a linear accelerator. Gross tumor volume with a $5 \mathrm{~cm}$ cranio-caudal and $2 \mathrm{~cm}$ radial margin received up to 50.4 Gy in 28 daily 1.8 Gy fractions 5 days a week, using anterior and posterior opposed fields for first 30.6 Gy in 18 fractions followed by three field technique (one anterior and two posterior oblique beams) for the remaining 10 fractions.

Concurrent chemotherapy was planned for all the patients only during external radiotherapy consisting of intravenous cisplatin $100 \mathrm{mg} / \mathrm{m}^{2}$ in the first and last 3 days of EBRT (i.e. 2 courses with 3-week interval), and oral capecitabine $825 \mathrm{mg} / \mathrm{m}^{2}$ twice daily for 28 days. Chemotherapy was withheld if the patient developed any complications such as nephrological or hematological toxicities, and restarted if/once the patient improved. None of the patients received chemotherapy prior to or concurrent with brachytherapy or neo/adjuvant to EBRT.

\section{Patient evaluation during treatment}

Subjects were visited weekly during the treatment, and were asked and examined of any treatment related toxicity. Blood tests were collected weekly in order to check for anemia, thrombocytopenia, and neutropenia. To assess the efficacy of treatment on patients' quality of life, we utilized the validated Persian translated version of EORTC-QLQ-C30 instrument in three times before (pre-brachytherapy), during (before EBRT), and after treatment (one week after completion of EBRT). Dysphagia was assessed by EOS18 instrument in 8 times including before $1^{\text {st }}$ and $2^{\text {nd }}$ brachytherapy sessions, before CRT, $2^{\text {nd }}$ week of CRT, at completion of treatment, one, two and three months after treatment. Additionally, patients reported dysphagia was graded based on $\mathrm{WHO}$ criteria as follows: 0 - normal diet, 1 - able to eat solid foods, 2 - able to eat semi-solid food, 3 - just able to drink, 4 - complete obstruction. The treatment tumor response was evaluated by surgical specimen or CT/upper gastrointestinal (GI) endoscopy if patients did not undergo surgical resection.

\section{Post-treatment follow-up}

After completion of treatment, the patients were followed-up, during which all patients underwent esophago- 
Table 1. Patient and tumor characteristics

\begin{tabular}{lc}
\hline Gender ratio (M/F) & $1.85: 1$ \\
\hline Age (mean, range) [years] & $66(42-81)$ \\
\hline T stage & $12 / 20$ \\
\hline T3 & $4 / 20$ \\
\hline N staging & $4 / 20$ \\
\hline Negative & $16 / 20$ \\
\hline Positive & $4 / 20$ \\
\hline Tumor location & $16 / 20$ \\
\hline Middle thoracic & \\
\hline Distal thoracic & $17 / 20$ \\
\hline Histology & $3 / 20$ \\
\hline Squamous cell carcinoma & $3(3-6)$ \\
\hline Adenocarcinoma & \\
\hline Follow-up time [months] &
\end{tabular}

gastroduodenoscopy. At this step, treatment response was assessed according to WHO criteria: a complete response (CR) defined as no macroscopic tumor; near complete remission was defined as a residue of only a few millimeters in diameter that detected by endoscopy; a partial remission (PR) occurred when at least $50 \%$ tumor reduction was found; no change (NC) in case of variation within $50 \%$ regression and $25 \%$ progression of the tumor; progressive disease was defined as an increase of tumor size by at least $25 \%$.

The radiotherapy treatment data was collected from the radiotherapy records of the patient. The initial length, volume, eccentricity, etc., of the gross tumor at presentation was identified from the planning CT images on the treatment planning system (RT dose plan). The other relevant data was collected from the medical records of the patient. The outcome parameters were calculated from the date of completion of treatment. We recorded any details of the patient having developed complications. The date and status at last follow-up were noted.

The collected data was analyzed using Statistical Package for the Social Sciences (SPSS) for Windows Version 21 (SPSS Inc; Chicago, IL, USA). In all analytical tests, $p<0.05$ was considered as statistically significant.

\section{Results}

Twenty patients completed full treatment course at our institute between 2013 and 2014. Patients' mean age was $66 \pm 10.5$ years (range: $42-81$ ) and $65 \%$ of them were male (Table 1). The reported pathology was squamous cell and adenocarcinomas in $85 \%$ and $15 \%$, respectively. In $80 \%$ of patients, the tumor was located in lower thoracic esophagus $(30-40 \mathrm{~cm})$ while the remaining was found in middle thoracic esophagus $(25-30 \mathrm{~cm})$. Twenty percent of our patients were node negative and the remaining were all node positive T3 $(12,60 \%)$ or T4 $(4,10 \%)$.

\section{Treatment response}

The median follow-up duration was 3 months. Three patients underwent surgical resection following chemoradiation (Table 2). Of these, one had complete pathologic response (TON0), one had moderate response (T2N0), and the other had poor response (T2N1). Among non-operated patients, $100 \%$ responded completely or partially to the treatment according to endoscopic findings 3-month post-treatment. In addition, considering both operable and non-operable subjects, 15 out of 17 tumors with squamous histology and 1 of 3 with adenocarcinomas experienced complete response. The rate of complete response was $75 \%$ and $81.25 \%$ in middle and distal thoracic locations, respectively. According to post treatment CT scan findings, the esophagus was normal in $13(65 \%)$ subjects. Similar to upper GI endoscopy, complete clinical response was more pronounced in squamous cell carcinoma (SCC) (vs. adenocarcinoma) and distal (vs. middle) thoracic tumors.

\section{Symptoms}

One month after completion of treatment, 4 patients had residual disease, which was identified on evaluation for persistent dysphagia. According to ANOVA test for repeated measures, the scores of dysphagia showed significant improvement from $1^{\text {st }}$ (before brachytherapy) to $8^{\text {th }}$ (3 month after completion of external radiotherapy) measurement $(F(7,152)=7.807, p<0.001)$. A Tukey posthoc test revealed that there was statistically significant difference in dysphagia between first (before brachytherapy) and third (after completion of chemoradiation) scoring. There was a statistically significant difference in deglutition $(p=0.002)$, eating scores $(p<0.001)$, pain $(p=0.001)$, GI symptom $(p<0.001)$ scores in 8 questionnaires as determined by one-way ANOVA. A Tukey post-hoc test revealed that there was statistically significant difference before start of brachytherapy (first questionnaire) and be-

Table 2. The characteristics of patients undergoing esophagectomy

\begin{tabular}{ccccccc} 
Patient & Age [years] & Gender & Histology & Location & Initial TNM staging & Surgical TNM staging \\
\hline 1 & 64 & Male & SCC & Distal thoracic & T3N1MO & T2N1MO \\
\hline 2 & 62 & Female & SCC & Distal thoracic & T3NOMO & TONOMO \\
\hline 3 & 47 & Male & SCC & Distal thoracic & T3N2MO & T2NOMO
\end{tabular}

SCC - squamous cell carcinoma 
fore start of chemoradiation (second questionnaire), and advanced course compared to the beginners' course.

The intensity of dysphagia was recorded according to WHO criteria as well (Table 3). After completion of treatment, none of the patients experienced grade 3 or more dysphagia and about one fifth reported grade 2 dysphagia, whereas $80 \%$ complained of grade 2 or 3 dysphagia.

There was no a statistically significant difference in quality of life (QoL) scores among 3 questionnaires as determined by one-way ANOVA $(p=0.939)$.

\section{Treatment toxicity}

The treatment was well tolerated by the patients. One patient developed esophageal perforation during second brachytherapy insertion, and underwent surgery shortly. No grade 4 toxicity was recorded on follow-up. Only one patient experienced grade 3 hematologic toxicity. Eleven patients (55\%) developed grade 1 (10 patients) or 2 (one patient) anemia, 9 (45\%) developed thrombocytopenia (5 grade 1, 3 grade 2, and one grade 3 ). None of the patients complained of dehydration, significant weight loss, grade 3 or 4 mucositis, necrosis, pneumonitis, or bleeding during and after treatment.

\section{Co-interventions}

None of the study participants underwent balloon or stent dilatation and gastrojejunostomy. Two patients received transient parenteral nutrition for 3 days. No food supplements were allowed during treatment. All of the patients received antiemetic (due to cisplatin emetogenicity).

\section{Discussion}

Surgery by itself was understood as the standard treatment for esophageal cancer for decades. In the past 30 years, numerous clinical trials have tested the efficacy of adding neoadjuvant or adjuvant radiochemotherapy to surgery $[9,10,11,12]$. These efforts, reflected in meta-analyses, introduced neoadjuvant radiochemotherapy as a new standard of care throughout the world $[13,14]$. The most important and annoying symptom of esophageal cancer is dysphagia presenting in over $70 \%$ of patients. This problem may lead to malnutrition, weight loss, fatigue, and treatment intolerance. In addition, $75 \%$ of patients experience transient exacerbation of dysphagia during the treatment. Thus, a critical issue before the initiation of neoadjuvant treatment is maintaining an appropriate nutritional state by removing the alimentary tube blockade or providing nutritional access (e.g. jejunostomy). There are some ways to alleviate dysphagia including laser therapy, external radiotherapy (EBRT), chemotherapy, endoluminal stent, and brachytherapy [15]. The latter two are the most commonly used methods. In two large RCTs, it was revealed that although stent provide more rapid resolution of food passage blockade but the effect of brachytherapy was more durable with less redilatation maneuvers and better quality of life $[16,17]$. Most studies of this kind have discussed incurable (and usually inoperable) patients $[18,19]$. However, in curable patients, endoluminal stents insertion before neoadjuvant chemoradiotherapy
Table 3. Number of patients experiencing highest grade of dysphagia before brachytherapy and after chemoradiation based on WHO criteria

\begin{tabular}{lcc}
$\begin{array}{l}\text { Grade } \\
\text { of dysphagia }\end{array}$ & $\begin{array}{c}\text { Pre-treatment } \\
\text { count [\%] }\end{array}$ & $\begin{array}{c}\text { Post-treatment } \\
\text { count [\%] }\end{array}$ \\
\hline 0 & - & $8(40)$ \\
\hline 1 & $4(20)$ & $8(40)$ \\
\hline 2 & $9(45)$ & $4(20)$ \\
\hline 3 & $7(35)$ & - \\
\hline 4 & - & -
\end{tabular}

make radiotherapy planning and dose distribution more difficult, and seems to be not preferred in these settings. In contrast, brachytherapy may reduce the tumor volume providing potential better response to external chemoradiation and fewer complications due to reduced filed size.

Several studies have shown improved local control and durable alleviation of dysphagia by adding brachytherapy to EBRT in esophageal cancers $[19,20,21]$. The only existent randomized controlled trial in this context showed the superiority of brachytherapy over EBRT boost [22]. In majority of literature, brachytherapy boost was added following EBRT not prior to it $[22,23,24]$. In present study, we tested the role of brachytherapy in reducing tumor volume and alleviation of dysphagia prior to standard chemoradiation with curative intention. In addition, by starting with brachytherapy we could better recognize the tumor location compared to post-EBRT when usually no tumor is visible [4]. In metastatic disease and poor performance state, the combination of EBRT and brachytherapy is intolerable; therefore, stents are preferred in such settings [25]. Accordingly, we only accrued high performance and potentially operable patients.

The results of our study were expectedly promising. All the patients more or less experienced resolution of dysphagia while $80 \%$ showed complete clinical response, which is an important predictor of survival [26]. The toxicity profile was acceptable. Pain was reduced in some patients but few experienced augmented pain due to radiation induced ulcers.

Considering small sample size and different design, it is difficult to compare our results with literature. In fact, we did not aim to compare outcomes but to test the safety and feasibility of performing brachytherapy prior to chemoradiation in potentially operable patients. The majority of previous studies used brachytherapy as a boost to tumor bed after EBRT. Other investigators have opted different prescribed dose, technology, and treatment field designs $[6,18,19,20,22,27]$. Another issue to be pointed out is that in other studies adenocarcinomas were dominant histology [28] or at least equal to squamous cell carcinomas [18]. In our study, SCC severely predominate adenocarcinoma. This is partly because SCC is more common in Iran compared to western countries. Furthermore, we did not include patients with esophagogastric junction tumors, which are mostly of adenocarcinoma histology. This could explain promising response rates in our study as mentioned in results section we observed poorer outcomes with adenocarcinomas. 
One limitation of our study that may impact results interpretation is "what did cause dysphagia to resolve at 3 months after chemoradiation completion?". One could say that this is the combined effect of brachytherapy and EBRT. The exacerbation of dysphagia after two weeks of EBRT initiation apparently neutralized the effect of brachytherapy. We can defend the role of brachytherapy in alleviating dysphagia in our study on the basis of following statements: 1) many patients experienced relief from dysphagia few days after brachytherapy; 2) none of the subjects lost weight during treatment course; 3) the rate of complications during EBRT was less compared to studies with standard neoadjuvant treatment that could be due to better nutritional state of our patients; 4) overall rate of dysphagia resolution was more than standard neoadjuvant treatment trials; 5) no patient needed other intervention both during and after treatment to resolve dysphagia; these could explain the role of brachytherapy in alleviating dysphagia and better treatment tolerance.

Another limitation of our study is the low proportion of subjects undergoing surgical esophagectomy. Therefore, we failed to report perioperative mortality as one of our preliminary goals. We should say that the reason for not to undergo surgery was patients' preference not surgeons. Many patients did not accept the risk of surgery after resolution of symptoms and low perceived chance of cure. This decision could partly be explained by our cultural differences with western societies in those standard neoadjuvant protocols have been studied. In addition, our follow-up period was relatively shorter than earlier studies, so we could not assess long term complications and survival rate.

It is worth saying that during the study, we have faced sanctions that withheld brachytherapy source renewal, so our accrual closed earlier than planned. We recommend using a control group in further studies to compare the effect of brachytherapy in resolution of dysphagia in contrast to other methods like chemotherapy in operable patients.

\section{Conclusions}

Intraluminal brachytherapy was a feasible and safe procedure to induce rapid and durable relief from dysphagia effectively before external beam radiotherapy with concurrent chemotherapy.

\section{Acknowledgments}

This research was not supported by any financial institution. All the authors declare no industrial link. We thank our colleagues from Department of Radiation Oncology of Cancer Institute, Imam Khomeini Hospital Complex for their contribution in patients' accrual and enrollment.

\section{Disclosure}

Authors report no conflict of interest.

\section{References}

1. Jemal A, Bray F, Center MM et al. Global cancer statistics. $C A$ Cancer J Clin 2011; 61: 69-90.
2. Siegel R, Naishadham D, Jemal A. Cancer statistics, 2013. CA Cancer J Clin 2013; 63: 11-30.

3. Koike R, Nishimura Y, Nakamatsu K et al. Concurrent chemoradiotherapy for esophageal cancer with malignant fistula. Int J Radiat Oncol Biol Phys 2008; 70: 1418-1422.

4. Vuong T, Szego P, David M et al. The safety and usefulness of high-dose-rate endoluminal brachytherapy as a boost in the treatment of patients with esophageal cancer with external beam radiation with or without chemotherapy. Int J Radiat Oncol Biol Phys 2005; 63: 758-764.

5. Buzurovic IM, Hansen JL, Bhagwat MS et al. Clinical implementation of a novel applicator in high-dose-rate brachytherapy treatment of esophageal cancer. J Contemp Brachytherapy 2016; 4: 319-325.

6. Lettmaier S, Strnad V. Intraluminal brachytherapy in oesophageal cancer: defining its role and introducing the technique. J Contemp Brachytherapy 2014; 6: 236-241.

7. Homs MYV, Steyerberg EW, Eijkenboom WMH et al. Single-dose brachytherapy versus metal stent placement for the palliation of dysphagia from oesophageal cancer: multicentre randomised trial. Lancet 2004; 364: 1497-1504.

8. Gaspar LE, Nag S, Herskovic A et al. American Brachytherapy Society (ABS) consensus guidelines for brachytherapy of esophageal cancer. Int J Radiat Oncol Biol Phys 1997; 38: 127-132.

9. Le Prise E, Etienne PL, Meunier B et al. A randomized study of chemotherapy, radiation therapy, and surgery versus surgery for localized squamous cell carcinoma of the esophagus. Cancer 1994; 73: 1779-1784.

10. Minsky BD, Neuberg D, Kelsen DP et al. Final report of Intergroup Trial 0122 (ECOG PE-289, RTOG 90-12): phase II trial of neoadjuvant chemotherapy plus concurrent chemotherapy and high-dose radiation for squamous cell carcinoma of the esophagus. Int J Radiat Oncol Biol Phys 1999; 43: 517-523.

11. Stahl M, Wilke $H$, Stuschke $M$ et al. Clinical response to induction chemotherapy predicts local control and long-term survival in multimodal treatment of patients with locally advanced esophageal cancer. J Cancer Res Clin Oncol 2005; 131: 67-72.

12. Cooper JS, Guo MD, Herskovic A et al. Chemoradiotherapy of locally advanced esophageal cancer: long-term follow-up of a prospective randomized trial (RTOG 85-01). JAMA 1999; 281: 1623-1627.

13. Moehler M, Al-Batran SE, Andus T et al. German S3-guideline "Diagnosis and treatment of esophagogastric cancer". Z Gastroenterol 2011; 49: 461-531 [Article in German].

14. Sjoquist KM, Burmeister BH, Smithers BM et al. Survival after neoadjuvant chemotherapy or chemoradiotherapy for resectable oesophageal carcinoma: an updated meta-analysis. Lancet Oncol 2011; 12: 681-692.

15. Sreedharan A, Harris K, Crellin A et al. Interventions for dysphagia in oesophageal cancer. Cochrane Database Syst Rev 2009; (4): CD005048.

16. Laskar SG, Lewis S, Agarwal JP et al. Combined brachytherapy and external beam radiation: an effective approach for palliation in esophageal cancer. J Contemp Brachytherapy 2015; 6: 453-461.

17. Bergquist H, Wenger U, Johnsson E. Stent insertion or endoluminal brachytherapy as palliation of patients with advanced cancer of the esophagus and gastroesophageal junction. Results of a randomized, controlled clinical trial. Dis Esophagus 2005; 18: 131-139.

18. Taal BG, Aleman BM, Koning CC et al. High dose rate brachytherapy before external beam irradiation in inoperable oesophageal cancer. Br J Cancer 1996; 74: 1452-1457.

19. Hujala K, Sipilä J, Minn H et al. Combined external and intraluminal radiotherapy in the treatment of advanced oesophageal cancer. Radiother Oncol 2002; 64: 41-45. 
20. Rosenblatt E, Jones G, Sur RK et al. Adding external beam to intra-luminal brachytherapy improves palliation in obstructive squamous cell oesophageal cancer: a prospective multi-centre randomized trial of the International Atomic Energy Agency. Radiother Oncol 2010; 97: 488-494.

21. Fabrini MG, Perrone F, De Liguoro $M$ et al. A single-institutional brachytherapy experience in the management of esophageal cancer. Brachytherapy 2010; 9: 185-191.

22. Sur RK, Singh DP, Sharma SC et al. Radiation therapy of esophageal cancer: Role of high dose rate brachytherapy. Int J Radiat Oncol Biol Phys 1992; 22: 1043-1046.

23. Petrovich Z, Langholz B, Formenti S et al. Management of carcinoma of the esophagus: the role of radiotherapy. Am J Clin Oncol 1991; 14: 80-86.

24. Hyden EC, Langholz B, Tilden T et al. External beam and intraluminal radiotherapy in the treatment of carcinoma of the esophagus. J Thorac Cardiovasc Surg 1988; 96: 237-241.

25. Allum WH, Griffin SM, Watson A et al. Guidelines for the management of oesophageal and gastric cancer. Gut 2002; 50 (Suppl 5): 1-23.

26. Giorgetti A, Pallabazzer G, Ripoli A et al. Prognostic Significance of 2-Deoxy-2-[18F]-Fluoro-D-Glucose PET/CT in Patients With Locally Advanced Esophageal Cancer Undergoing Neoadjuvant Chemoradiotherapy Before Surgery. Medicine (Baltimore) 2016; 95: e3151.

27. Patonay P, Naszály A, Mayer A. Simultaneous radiochemotherapy and endoluminal HDR brachytherapy in esophageal cancer. Strahlenther Onkol 2007; 183: 94-98 [Article in German]

28. Aggarwal A, Harrison M, Glynne-Jones R et al. Combination external beam radiotherapy and intraluminal brachytherapy for non-radical treatment of oesophageal carcinoma in patients not suitable for surgery or chemoradiation. Clin Oncol (R Coll Radiol) 2015; 27: 56-64. 